\title{
Changes in Liver Gene Expression of Azin1 Knock-out Mice
}

\author{
Tao Wan ${ }^{\mathrm{a}}$, Yuan $\mathrm{Hu}^{\mathrm{a}}$, Wenlu Zhang ${ }^{\mathrm{a}}$, Ailong Huang ${ }^{\mathrm{a}}$, Ken-ichi Yamamura ${ }^{\mathrm{b}}$, and \\ Hua Tang ${ }^{\mathrm{a}, *}$ \\ a Key Laboratory of Molecular Infectious Diseases, Ministry of Education, \\ Chongqing Medical University, Chongqing 400016, China. Fax: +86-23-68 486780. \\ E-mail: tanghua86162003@yahoo.com.cn \\ b Department of Developmental Genetics, Institute of Molecular Embryology and \\ Genetics, Kumamoto University, Kumamoto 860 -0811, Japan \\ * Author for correspondence and reprint requests \\ Z. Naturforsch. 65c, 519-527 (2010); received January 5/March 3, 2010
}

The ornithine decarboxylase antizyme inhibitor (AZI) was discovered as a protein that binds to the regulatory protein antizyme and inhibits the ability of antizyme to interact with the enzyme ornithine decarboxylase (ODC). Several studies showed that the AZI protein is important for cell growth in vitro. However, the function of this gene in vivo remained unclear. In our study, we analyzed the transcriptional profiles of livers on the 19th day of pregnancy of $A$ zin 1 knock-out mice and wild-type mice using the Agilent oligonucleotide array. Compared to the wild-type mice, in the liver of Azin1 knock-out mice 1812 upregulated genes (fold change $\geq 2$ ) and 1466 downregulated genes (fold change $\leq 0.5$ ) were showed in the microarray data. Altered genes were then assigned to functional categories and mapped to signaling pathways. These genes have functions such as regulation of the metabolism, transcription and translation, polyamine biosynthesis, embryonic morphogenesis, regulation of cell cycle and proliferation signal transduction cascades, immune response and apoptosis. Real-time PCR was used to confirm the differential expression of some selected genes. Overall, our study provides novel understanding of the biological functions of AZI in vivo.

Key words: Microarray, Azin1 Knock-out Mice, Ornithine Decarboxylase

\section{Introduction}

The polyamines spermine, spermidine, and their precursor putrescine are natural organic compounds that play a crucial role in regulating fundamental cellular processes such as proliferation, differentiation, transformation, and apoptosis (Janne et al., 1991; Pegg et al., 1995; Bercovich and Kahana, 2004). Ornithine decarboxylase (ODC) is the key enzyme in the biosynthesis of polyamines, and its degradation constitutes an important regulatory mechanism that controls cellular polyamines (Bercovich and Kahana, 2004). Many researches have shown that increased ODC and polyamine levels are associated with increased cell proliferation, decreased apoptosis and expression of genes affecting tumour invasion and metastasis (Gilmour et al., 1987; Holtta et al., 1988; Childs et al., 2003). High levels of polyamine can induce the production of a protein termed antizyme (AZ) which binds and inactivates ODC,

Tao Wan and Yuan $\mathrm{Hu}$ contributed equally to this work. and subsequently targets it for rapid degradation by the 26S proteosome (Bercovich et al., 1989; Murakami et al., 1992). In addition to its role in regulating the ODC activity and degradation, AZ was also demonstrated to regulate the polyamine transport across the plasma membrane via a yet unknown mechanism (He et al., 1994; Suzuki et al., 1994; Sakata et al., 2000).

The endogenous antizyme inhibitor (AZI) was first discovered as a protein that binds to antizyme and inhibits several of its functions. AZI blocks the ability of AZ to promote ODC degradation and to inhibit the ODC enzymatic activity (Fujita et al., 1982). AZI shares $65 \%$ homology with ODC and conserves the majority of residues that are required for ODC activity, but AZI itself does not exhibit any known enzymatic activity. AZI binds AZ with high affinity preventing the formation of the AZ-ODC complex and consequently suppressing ODC degradation (Kim et al., 2006). The human AZI gene is located on chromosome 8q22.3, and amplification of this region is found in several tumours (Schaner et al., 2005). AZI is overexpressed in gastric tumours (Jung et al., 
2000) and is induced in cells and tissues following growth stimulation (Kim et al., 2006; Mangold, 2006). Increased expression of AZI can elevate the polyamine levels, increase the cell growth, and provoke cellular transformation (Keren-Paz et al., 2006).

Gene knock-out mice are a useful model to study the biological functions of the corresponding genes. In a mouse line named Ayu17-689 which was obtained using the gene trap technology, the trap vector pU-17 was inserted into the Azin1 gene (Tang et al., 2009). The homozygous mutant mice died on P0 (the day of birth) with abnormal liver morphology which resulted in degradation of ODC and reduced the biosynthesis of putrescine and spermidine. These results showed that AZI plays an important role in regulating the levels of ODC, putrescine, and spermidine, and is essential for the survival of mice (Tang et al., 2009). To identify the importance and biological functions of AZI in vivo, we analyzed the differences of gene expression profiles in the liver of Azin $^{+/+}$and $A$ zin $^{-/-}$mice using cDNA microarrays in the present study.

\section{Material and Methods}

\section{Animals}

Heterozygous Ayu17-689 mice $\left(A z i n 1^{+-}\right)$were produced in the Center for Animal Resources and Development, Kumamoto University, Japan, and fed in the Center of Experimental Animals, Chongqing Medical University, China. The mice were housed in polyethylene cages containing wood shavings and were given rodent chow and water ad libitum. Mice were housed in rooms at $24{ }^{\circ} \mathrm{C}$, a humidity of $55 \%$, and a 12 -h light/dark cycle. Five heterozygous males were mated with ten heterozygous females to produce wild-type mice, heterozygous mice, and homozygous mice. Because the homozygous mutant mice died on $\mathrm{P} 0$, heterozygous female mice were performed by abdominal delivery on the 19th day of pregnancy. The liver tissues of offspring mice were taken out and preserved in liquid nitrogen, then kept at $-80{ }^{\circ} \mathrm{C}$ until further analysis. At that time, tail biopsies were taken for genotyping.

\section{Extraction of total RNA}

Liver samples from 3 wild-type, 3 heterozygous, and 3 homozygous mice were pooled for each genotype. Each pool was placed into $3 \mathrm{~mL}$ of
Trizol solution (Invitrogen, Carlsbad, USA) and homogenized with a polytron homogenizer. Total RNA was separated with Qiagen Rneasy mini kit (Qiagen, Hilden, Germany), according to the protocol described by the manufacturer, including a DNase digestion step. RNA quantification and quality assurance were assessed by analyzing the A260/A280 ratio (between 1.8 and 2.1) using a spectrophotometer, and RNA integrity and genome DNA contamination were tested by denaturing agarose gel electrophoresis.

\section{DNA microarray}

The RNA samples were amplified and labeled using the Agilent Quick Amp labeling kit (Agilent Technologies, Santa Clara, CA, USA) according to the manufacturer's instructions and hybridized with the Agilent whole genome oligonucleotide microarray in Agilent's SureHyb hybridization chambers. After hybridization and washing, the processed slides were scanned with the Agilent DNA microarray scanner (part number G2505B) using settings recommended by Agilent Technologies. The procedure above was completed by KangChen Bio-tech (Shanghai, China).

\section{Microarray data processing}

The resulting text files extracted from Agilent Feature Extraction Software (version 9.5.3) were imported into the Agilent GeneSpring GX software (version 7.3 or later) for further analysis. The microarray data sets were normalized in GeneSpring GX using the Agilent FE one-colour scenario (mainly median normalization), and genes marked present were chosen for data analysis. Differentially expressed genes were identified through fold-change screening. The profiling identified a subset of the total number of probes analyzed by the Agilent whole genome oligo microarray that are differentially expressed. GO (gene ontology) analysis and pathway analysis were performed on this subset of genes.

\section{Real-time PCR}

Differentially expressed genes of interest were selected for validation of the microarray results by real-time PCR. Total RNA from liver tissues, $A z i n 1^{+/+}$or $A z i n 1^{-/-}$mice, were prepared as described above. $2 \mu \mathrm{g}$ of total RNA (the same RNA used for the arrays) were reverse transcribed using M-MLV Reverse Transcriptase (Promega, 
Table I. Primers used for real-time PCR.

\begin{tabular}{llll}
\hline Gene & $\begin{array}{l}\text { Span of } \\
\text { bases }\end{array}$ & Forward primer & Reverse primer \\
\hline LOC385413 & $120-174$ & 5'-GGCCACCATGCCAAGGA-3' & 5'-GCGGCGACCTGAAGACTCT-3' \\
Nup98 & $1834-1897$ & 5'-CAGGCACAGCCAAATCACAT-3' & 5'-CCGTTGGCTAGAGATGGTTCA-3' \\
Olfr1351 & $238-302$ & 5'-CCACAGATGCTGGTGACCATT-3' & 5'-TGGGTGATGCAGTTTGCATAG-3' \\
Ace & $1964-2034$ & 5'-GGGCATTGACCTAGAGACTGATG-3' & 5'-CTTGGGCTGTCCGGTCATAC-3' \\
Trpd52 13 & $636-693$ & 5'-CAGTGGCCTTCTCCCTTCAC-3' & 5'-ACTCCCCAGAGCCGAGTGT-3' \\
Pvrl4 & $3019-3079$ & 5'-CATGGCCCCTCGAATCTTG-3' & 5'-CCAACGCCGTGAAGGTACA-3' \\
Atp8a1 & $3629-3687$ & 5'-CAGAGCCTATGACACCACGAAA-3' & 5'-GGCTTAGGCCTGCCAATCA-3' \\
Defb7 & $61-123$ & 5'-CATTTGCAGCTTTTAGCCAAGA-3' & 5'-ATTCGCCTCCTTCCCGATA-3' \\
Dner & $1388-1538$ & 5'-ACACTTGTGGTGAAGGCTAG-3' & 5'-CTTCACAGAAGGTGCCAATG-3' \\
Gng3 & $351-471$ & 5'-ATAAAGGTGTCCAAGGCAGC-3' & 5'-AGAAGAACTTCTTCTCCCGG-3' \\
Elavl3 & $588-813$ & 5'-TTCAAGAGTCTCTTCGGCAG-3', & 5'-TGACATACAGGTTGGCATCC-3' \\
Igfbpl1 & $640-806$ & 5'-TATAGCTGTCCAGGTGCGAG-3', & 5'-ATCTGTTCAGATCGAGGACC-3' \\
Mapk10 & $841-1041$ & 5'-TTAAGAAGCTCAGCAGACCC-3' & 5'-TCTGAATCACCTGACACAGG-3' \\
Pga5 & $382-531$ & 5'-ATCTTCAGTCCTGTGGGTAC-3', & 5'-AGAAATCCGGACATCTCTCC-3' \\
Sst & $200-446$ & 5'-TTTCTGCAGAAGTCTCTGGC-3' & 5'-AGGATGTGAATGTCTTCCAG-3' \\
\hline
\end{tabular}

Madison, USA) with an oligo (dT) primer. Genespecific primers (Table I) were used to amplify message RNA by real-time PCR on the IQ5 multicolour real-time PCR detection system (BioRad, Hercules, CA, USA) using Power SYBR ${ }^{\circledR}$ Green PCR Master Mix (Applied Biosystems, Foster, CA, USA). Primer sets were designed using ABI Primer Express software, and the primer set of mouse GAPDH gene was designed as control. The optimal annealing temperature for each primer set was determined prior to the analysis of experimental samples. The efficiency of the PCR reaction for each primer set was determined using serial dilution of cDNA from mouse liver as described above. Triplicate $25 \mu \mathrm{L}$ real-time PCR reactions were run, containing $12.5 \mu \mathrm{L}$ SYBR Green PCR master mix, $1 \mu \mathrm{L}$ of a primer stock solution containing $10 \mu \mathrm{M}$ of both forward and reverse primers, $1 \mu \mathrm{L}$ of cDNA template, and 10.5 $\mu \mathrm{L}$ nuclease-free water. The following standard PCR conditions were used: one cycle at $90{ }^{\circ} \mathrm{C}$ for $10 \mathrm{~min}$ and 40 cycles of $94^{\circ} \mathrm{C}$ for $15 \mathrm{~s}$, the primerspecific annealing temperature for $40 \mathrm{~s}$ (because different primers were used, every primer had its suitable annealing temperature, e.g., $58^{\circ} \mathrm{C}, 59^{\circ} \mathrm{C}$, $60{ }^{\circ} \mathrm{C}$; here we described this process as primerspecific annealing temperature), and $72{ }^{\circ} \mathrm{C}$ for 60 s. A cycle threshold (CT) was assigned at the beginning of the logarithmic phase of PCR amplification, and duplicate CT values were analyzed by Microsoft Excel. The $2^{-\Delta \Delta \mathrm{Ct}}$ method (Livak and Schmittgen, 2001) was used to determine the relative expression of the gene in each sample.

\section{Results}

\section{Microarray analysis}

Microarray analysis was done to determine the hepatic gene expression between $A z i n 1^{+/+}$and $A z i n 1^{-/}$mice. As compared with $A z i n 1^{+/+}$mice, the expression of 3278 genes (fold change $\geq 2$ ) was changed in $A z i n 1^{-1}$ mice; 1812 genes were upregulated, while 1466 genes were downregulated. The scatter plot analysis of all genes identified during microarray expression profiling is showed in Fig. 1. The genes strongly increased (fold change $\geq 10$ ) in Azin1 $1^{-/}$knock-out mice compared with $A z$ in $1^{+/+}$ mice are Itgb3, Wdr40c, Fgl1, Bcorl1, Stag2, Ifit1, Olfr1495, Amd1, Tcfcp2 11, Vcpip1, Nfia, Map2k3, Hmgb1, and Chek1 (Table II). Meanwhile, the genes greatly decreased (fold change $<0.05$ ) in Azin $1^{-1-}$ knock-out mice compared with $A z i n 1^{+/+}$ mice are Pga5, Stmn2, Ncan, Nsg2, Trim67, Stmn3, Zcchc12, Dner, B3gat1, Agr2, Sst, Gng3, Mapt, U46068, Fez1, and Elav13 (Table III).

\section{Gene ontology analysis}

All different expression genes were mapped to specific biological processes by GO analysis. For the sake of clarity, related GO terms were combined to twelve superordinate categories (Fig. 2). Most significant genes were mapped to the biological process of cellular morphogenesis (cellular morphogenesis during differentiation, regulation of cell growth, cell development, and system development), metabolism (polyamine biosynthesis, 


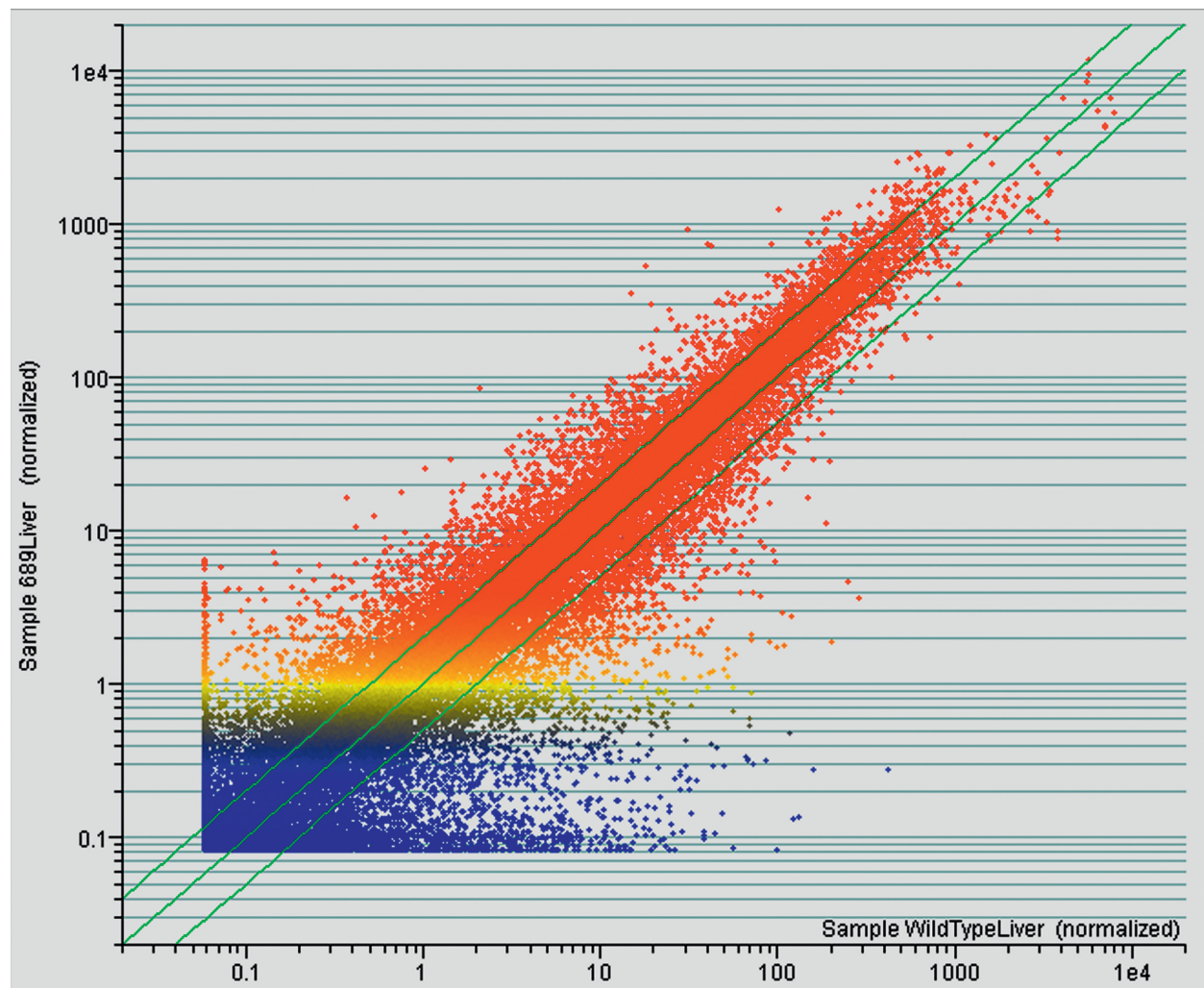

Fig. 1. Scatter plot analysis of all genes identified during microarray expression profiling. Cy3-labeled cDNA was amplified from the total RNA extracted from liver tissue of $A z i n 1^{+/+}$and $A z i n 1^{-/-}$mice. Data was analyzed using Agilent GeneSpring GX software. Individual genes (41,000+ present with good quality flags) were normalized to the median intensity of the array. Each spot is the mean value of an individual gene from two arrays. The outer green lines indicate the two-fold differential expression levels. There were 3278 genes with more than two-fold expression difference. Red, high expression; yellow, medium expression; blue, low expression.

polyamine metabolism, spermine biosynthesis/ metabolism, spermidine biosynthesis/metabolism, and other cellular molecule biosyntheses/metabolisms), and cellular physiological process (protein transport, cell cycle, cell proliferation, cell organization and biogenesis, regulation of growth).

\section{Pathway analysis}

In order to elucidate whole chains of events observed in our microarray experiment, pathway analysis was performed to map the list of significantly regulated genes in Azin1 $^{-/-}$mice liver compared with $A$ zin $1^{+/+}$mice liver. As expected, different expression genes were signed to several classes such as mRNA processing [mouse (Mus musculus $)$ ] $(p=4.97 \mathrm{E}-16)$, oxidative phosphorylation (Mus musculus) ( $p=1.1 \mathrm{E}-06)$, MAPK signaling pathway (Mus musculus) ( $p=0.00935)$, ubiquitin-mediated proteolysis (Mus musculus) $(p=0.00019)$, Wnt signaling pathway $(p=3.97 \mathrm{E}-$ 05), ribosome (Mus musculus) ( $p=4 \mathrm{E}-21)$, cytoplasmic ribosomal proteins $(p=8.99 \mathrm{E}-20)$, translation factors $(p=1.56 \mathrm{E}-08)$, electron transport chain ( $p=2.54 \mathrm{E}-06)$, cell cycle (Mus musculus) $(p=0.000367)$, amino-acid metabolism $(p=$ $0.00875)$, PPAR signaling pathway $(p=0.000878)$, DNA replication $(p=0.00278), \mathrm{G} 1$ to $\mathrm{S}$ cell cycle control ( $p=0.0108)$, purine metabolism (Mus musculus $)(p=0.00612)$ and so on.

\section{Real-time PCR}

Genes significantly changed in the arrays between $A$ zin $1^{+/+}$and $A$ zin $1^{I_{-}-}$mice liver were selected for verification by real-time PCR, including 8 
Table II. Genes intensively increased in $A z i n 1^{-/-}$mice in comparison with $A z i n 1^{+/+}$mice.

\begin{tabular}{llll}
\hline Genbank & Gene symbol & Fold change & Description \\
\hline NM_016780 & Itgb3 & 41.2 & Integrin beta 3 \\
NM_175539 & Wdr40c & 30.58 & WD repeat domain 40C \\
AK083397 & Fgl1 & 30.4 & Fibrinogen-like protein 1 \\
AK031119 & Bcorl1 & 23.77 & BCL6 co-repressor-like 1 \\
AK077318 & Stag2 & 15.49 & Stromal antigen 2 \\
NM_008331 & Ifit1 & 12.13 & Interferon-induced protein with tetratricopeptide repeats 1 \\
NM_146344 & Olfr1495 & 11.89 & Olfactory receptor 1495 \\
Z14986 & Amd1 & 11.47 & S-Adenosylmethionine decarboxylase 1 \\
AK086294 & Tcfcp2 11 & 11.33 & Transcription factor CP2-like 1 \\
NM_173443 & Vcpip1 & 11.31 & Valosin-containing protein (p97)/p47 complex interacting \\
& & & protein 1 \\
NM_010905 & Nfia & 11.26 & Nuclear factor I/A \\
NM_008928 & Map2k3 & 10.95 & Mitogen-activated protein kinase kinase 3 \\
NM_010439 & Hmgb1 & 10.62 & High-mobility group box 1 \\
NM_007691 & Chek1 & 10.29 & Checkpoint kinase 1 homologue $(S$. pombe $)$
\end{tabular}

Table III. Genes intensively decreased in $A z i n 1^{-/-}$mice in comparison with $A z i n 1^{+/+}$mice.

\begin{tabular}{llll}
\hline Genbank & Gene symbol & Fold change & Description \\
\hline NM_021453 & Pga5 & 0.00067 & Pepsinogen 5, group I \\
NM_025285 & Stmn2 & 0.00083 & Stathmin-like 2 \\
NM_007789 & Ncan & 0.00103 & Neurocan \\
NM_008741 & Nsg2 & 0.00111 & Neuron-specific gene family member 2 \\
BC094596 & Trim67 & 0.00151 & Tripartite motif-containing 67 \\
NM_009133 & Stmn3 & 0.00174 & Stathmin-like 3 \\
NM_028325 & Zcchc12 & 0.00247 & Zinc finger, CCHC domain containing 12 \\
NM_152915 & Dner & 0.00256 & Delta/notch-like EGF-related receptor \\
NM_029792 & B3gat1 & 0.00267 & Beta-1,3-glucuronyltransferase1 (glucuronosyltransferase P) \\
NM_011783 & Agr2 & 0.00291 & Anterior gradient 2 (Xenopus laevis) \\
NM_009215 & Sst & 0.003 & Somatostatin \\
NM_010316 & Gng3 & 0.00313 & Guanine nucleotide binding protein (G protein), gamma 3 \\
& & & subunit \\
NM_010838 & Mapt & 0.00341 & Microtubule-associated protein tau \\
NM_153418 & U46068 & 0.0037 & cDNA sequence U46068 \\
NM_183171 & Fez1 & 0.00383 & Fasciculation and elongation protein zeta 1 (zygin I) \\
NM_010487 & Elav13 & 0.00414 & ELAV (embryonic lethal, abnormal vision, Drosophila)-like 3 \\
& & & (Hu antigen C) \\
\hline
\end{tabular}

upregulated genes and 7 downregulated genes in Azin $1^{-1-}$ mice liver. The results are shown in Fig. 3. The majority of microarray data could be confirmed by real-time PCR. However, in 3 of the 15 genes, the real-time PCR data did not correlate with microarray data. Two genes, Nup98 and Atp8a1, which increased in $A$ zin $1^{-1-}$ mice liver over 10-fold compared with $A z i n 1^{+/+}$mice liver in the microarray showed no evident changes between these two types mice (Fig. 3a). The Pga5 gene which was downregulated in $A z i n 1^{-/}$mice liver in the microarray showed an opposite result in realtime PCR, Pga5 was a little higher expressed in Azin1 $1^{-/-}$mice liver compared with $A z$ in $1^{+++}$mice liver (Fig. 3b).

\section{Discussion}

The gene knock-out mice are important for us to study the biological functions of the corresponding genes. Using gene trap technology, we have generated the AZI knock-out mice. Because the homozygous mutant mice $\left(A z i n 1^{--}\right)$died on P0, we choose $A z i n 1^{1^{--}}$mice and $A z i n 1^{+/+}$mice from E19 (embryonic day) whose development has elementary matured. By microarrays, we compared the differences of liver gene expression between $A z i n 1^{+/+}$and $A z i n 1^{-/-}$mice. 1812 genes (fold change $\geq 2$ ) were upregulated and 1466 genes (fold change $\leq 0.5$ ) were downregulated in Azin $1^{-/-}$mice liver. 


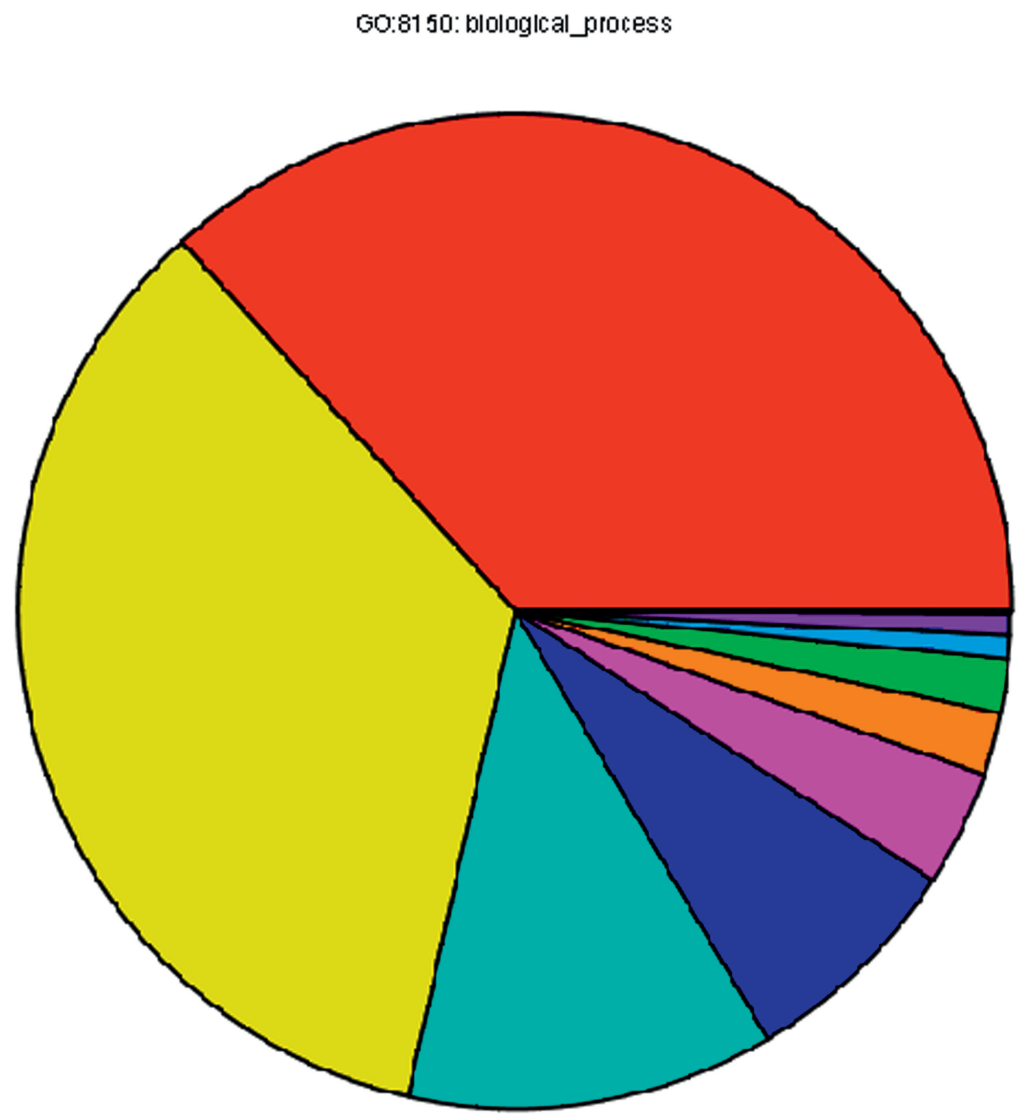

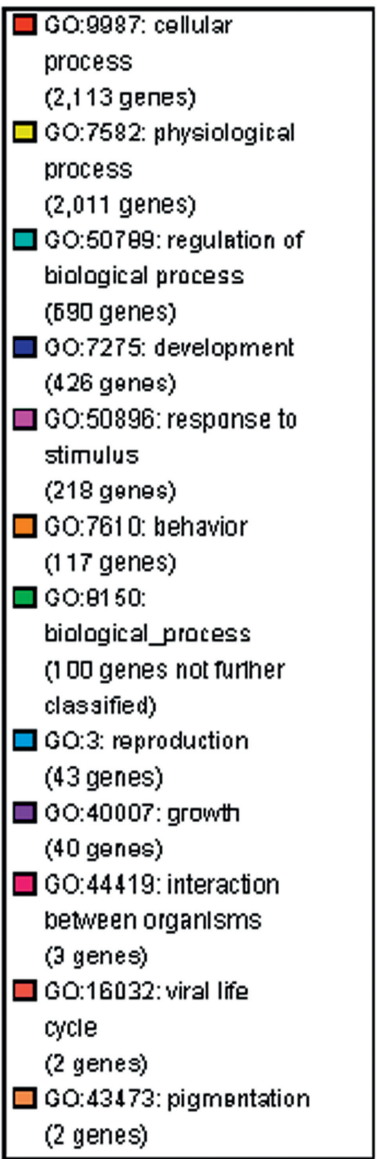

Fig. 2. Biological process identified by gene ontology (GO) mapping. Differentially expressed transcripts were mapped to numerous biological processes of the hierarchical GO system.

The results reported here suggest that the changes in the expression of many genes in $A$ zin $1^{-1-}$ mice liver reflect both direct and indirect effects of Azin1. AZ binds directly to ODC, and the AZ-ODC complex is degraded by the $26 \mathrm{~S}$ proteasome. AZI has a higher affinity for $\mathrm{AZ}$ than for ODC, and as such can displace ODC from the ODC-AZ complex and prevent ODC from being degraded by the $26 \mathrm{~S}$ proteasome (Kitani and $\mathrm{Fu}-$ jisawa, 1989). Therefore, AZI plays an important role in regulating the cellular polyamine metabolism. In our study, GO analysis of all different expression genes between $A z i n 1^{+/+}$and $A z i n 1^{-1-}$ mice liver showed that 5 of 11 genes associated with polyamine biosynthesis (GO: 6596, $p=0.00268$ ) were changed, and genes having functions of spermine biosynthesis (GO: 6597) and spermidine metabolism (GO: 8216) also changed in $\mathrm{Azin1}^{-1-}$ mice compared with $A z i n 1^{+++}$mice. The Amd1 gene encoding $S$-adenosylmethionine decarboxylase (AdoMetDC) was up-regulated over 10-fold in $A$ zin $1^{--}$mice liver. AdoMetDC is one of the key enzymes involved in the biosynthesis of spermidine and spermine and plays an essential role in embryonic development and cell proliferation (Nishimura et al., 2002). The increased expression of $A m d 1$ induced by knock-out of $A z$ in 1 indicated that Azin1 is important in the metabolism of polyamine and mouse embryonic development.

AZI has AZ-independent effects and binds directly to cyclin D1 to regulate the cell cycle. AZI overexpression results in increased cell growth as 

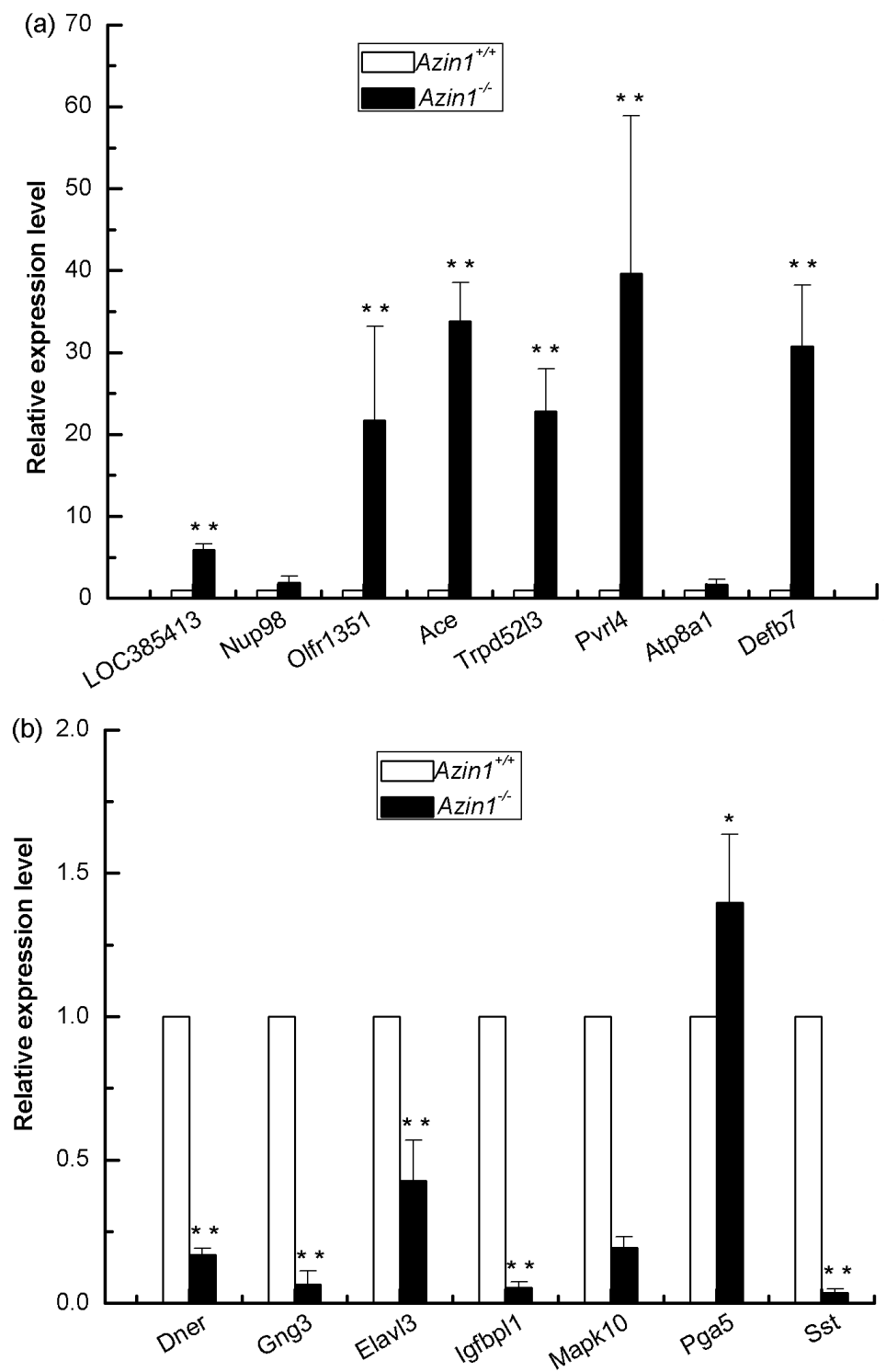

Fig. 3. Validation of microarray results by real-time PCR. (a) Expression of 8 genes upregulated in $A z i n 1^{-/-}$mice liver. (b) Expression of 7 genes downregulated in $A z i n 1^{-/}$mice liver. (** $p<0.001$; * $p<0.05$; $t$-test.)

well as the induction of the transformed phenotype (Kim et al., 2006). Compared with normal tissue, AZI is upregulated in gastric cancer as well as several other cancers (Jung et al., 2000; Rhodes et al., 2004). These results demonstrate that AZI is an important means of modulating cell proliferation and oncogenesis. Our study showed that two genes, Stag2 (Prieto et al., 2002) and Chek1 which have cell cycle functions, were upregulat- ed over 10 -fold in Azin1 $1^{-1-}$ mice liver. Chek1 is a conserved kinase that imposes cell cycle delays in response to impediments of DNA replication. Moreover, Chek1 appears to help defend genomic integrity through effects on several pathways, including Fanconi anemia proteins, the mitotic spindle, and transcription of cell cycle-related genes (Enders, 2008). This upregulation of Chek1 induced by Azin1 knock-out in mice liver may be 
associated with the development and embryonic lethality. Besides, two genes associated with cell proliferation and differentiation, Dner (Tohgo et al., 2006) and Elav13, were downregulated over 10-fold in Azin1 $1^{-1-}$ mice, and the Agr2 gene which was reported to promote tumour growth, cell migration, and cellular transformation (Wang et al., 2008) also decreased the expression over 100 -fold in $A z$ in $^{-/-}$mice. AZ has many important functions such as inducing apoptosis, regulating transcription, and promoting DNA repair (Tsuji et al., 2007; Liu et al., 2006). AZI can bind to AZ and may contribute to regulate these functions. Our microarray results showed that seven genes involved in regulation of transcription changed intensively in $A z i n 1^{-/}$mice compared with $A z$ in $1^{+/+}$mice. Four genes, Bcorl1, Tcfep2 11, Nfia (Shu et al., 2003), Hmgb1 were upregulated in $A z$ in $^{-1-}$ mice, and three genes, Dner, Sst, Fezl,

Bercovich Z. and Kahana C. (2004), Degradation of antizyme inhibitor, an ornithine decarboxylase homologous protein is ubiquitin-dependent and is inhibited by antizyme. J. Biol. Chem. 279, 54097-54102.

Bercovich Z., Rosenberg-Hasson Y., Ciechanover A., and Kahana C. (1989), Degradation of ornithine decarboxylase in reticulocyte lysate is ATP-dependent but ubiquitin-independent. J. Biol. Chem. 264, 15949-15952.

Childs A. C., Mehta D. J., and Gerner E. W. (2003), Polyamine-dependent gene expression. Cell. Mol. Life Sci. 60, 1394-1406.

Enders G. H. (2008), Expanded roles for Chk1 in genome maintenance. J. Biol. Chem. 283, 17749-17752.

Fujita K., Murakami Y., and Hayashi S. (1982), A macromolecular inhibitor of the antizyme to ornithine decarboxylase. Biochem. J. 204, 647-652.

Gilmour S. K., Verma A. K., Madara T., and O'Brien T. G. (1987), Regulation of ornithine decarboxylase gene expression in mouse epidermis and epidermal tumors during two-stage tumorigenesis. Cancer Res. 47, 1221-1225.

He Y., Suzuki T., Kashiwagi K., and Igarashi K. (1994), Antizyme delays the restoration by spermine of growth of polyamine-deficient cells through its negative regulation of polyamine transport. Biochem. Biophys. Res. Commun. 203, 608-614.

Holtta E., Sistonen L., and Alitalo K. (1988), The mechanisms of ornithine decarboxylase deregulation in cHa-ras oncogene-transformed NIH 3 T3 cells. J. Biol. Chem. 263, 4500-4507.

Janne J., Alhonen L., and Leinonen P. (1991), Polyamines: from molecular biology to clinical applications. Ann. Med. 23, 241-259. were downregulated in $A z i n 1^{-1-}$ mice. Meanwhile, the Mapt gene which is involved in apoptosis was downregulated over 100-fold in $A \operatorname{zin} 1^{-/}$mice. Besides, some other genes changed in $A$ zinl ${ }^{-1}$ mice indicated that AZI may has effect on the intracellular signaling cascade and immune response; more studies would be required to conclusively understand these.

Overall, the results of our study imply that the Azin1 gene is critical for the survival of mice. In addition, Azin1 plays an important role in the development of mice, the metabolism of polyamine, cell proliferation and differentiation, apoptosis and regulation of transcription.

\section{Acknowledgements}

This work was supported by Nature Science Foundation of China (30671080).

Jung M. H., Kim S. C., Jeon G. A., Kim S. H., Kim Y., Choi K. S., Park S. I., Joe M. K., and Kimm K. (2000), Identification of differentially expressed genes in normal and tumor human gastric tissue. Genomics 69, 281-286.

Keren-Paz A., Bercovich Z., Porat Z., Erez O., Brener O., and Kahana C. (2006), Overexpression of antizyme-inhibitor in NIH3T3 fibroblasts provides growth advantage through neutralization of antizyme functions. Oncogene 25, 5163-5172.

Kim S. W., Mangold U., Waghorne C., Mobascher A., Shantz L. M., Banyard J., and Zetter B. R. (2006), Regulation of cell proliferation by the antizyme inhibitor: evidence for an antizyme-independent mechanism. J. Cell Sci. 119, 2853-2861.

Kitani T. and Fujisawa H. (1989), Purification and characterization of antizyme inhibitor of ornithine decarboxylase from rat liver. Biochim. Biophys. Acta 991, 44-49.

Liu G. Y., Liao Y. F., Hsu P. C., Chang W. H., Hsieh M. C., Lin C. Y., Hour T. C., Kao M. C., Tsay G. J., and Hung H. C. (2006), Antizyme, a natural ornithine decarboxylase inhibitor, induces apoptosis of haematopoietic cells through mitochondrial membrane depolarization and caspases' cascade. Apoptosis 11, $1773-1788$.

Livak K. J. and Schmittgen T. D. (2001), Analysis of relative gene expression data using real-time quantitative PCR and the 2(-delta delta C(T)) method. Methods 25, 402-408.

Mangold U. (2006), Antizyme inhibitor: mysterious modulator of cell proliferation. Cell. Mol. Life Sci. 63, 2095-2101. 
Murakami Y., Matsufuji S., Kameji T., Hayashi S., Igarashi K., Tamura T., Tanaka K., and Ichihara A. (1992), Ornithine decarboxylase is degraded by the 26S proteasome without ubiquitination. Nature 360, 597-599.

Nishimura K., Nakatsu F., Kashiwagi K., Ohno H., Saito T., and Igarashi K. (2002), Essential role of $S$-adenosylmethionine decarboxylase in mouse embryonic development. Genes Cells 7, 41-47.

Pegg A. E., Shantz L. M., and Coleman C. S. (1995), Ornithine decarboxylase as a target for chemoprevention. J. Cell Biochem. 22, 132-138.

Prieto I., Pezzi N., Buesa J. M., Kremer L., Barthelemy I., Carreiro C., Roncal F., Martinez A., Gomez L., Fernandez R., Martinez A. C., and Barbero J. L. (2002), STAG2 and Rad21 mammalian mitotic cohesins are implicated in meiosis. EMBO Rep. 3, 543-550.

Rhodes D. R., Yu J., Shanker K., Deshpande N., Varambally R., Ghosh D., Barrette T., Pandey A., and Chinnaiyan A. M. (2004), ONCOMINE: a cancer microarray database and integrated data-mining platform. Neoplasia 6, 1-6.

Sakata K., Kashiwagi K., and Igarashi K. (2000), Properties of a polyamine transporter regulated by antizyme. Biochem. J. 347, 297-303.

Schaner M. E., Davidson B., Skrede M., Reich R., Florenes V. A., Risberg B., Berner A., Goldberg I., Givant-Horwitz V., Trope C. G., Kristensen G. B., Nesland J. M., and Borresen-Dale A. L. (2005), Variation in gene expression patterns in effusions and primary tumors from serous ovarian cancer patients. Mol. Cancer 4, 26.

Shu T., Butz K. G., Plachez C., Gronostajski R. M., and Richards L. J. (2003), Abnormal development of forebrain midline glia and commissural projections in Nfia knock-out mice. J. Neurosci. 23, 203-212.

Suzuki T., He Y., Kashiwagi K., Murakami Y., Hayashi S., and Igarashi K. (1994), Antizyme protects against abnormal accumulation and toxicity of polyamines in ornithine decarboxylase-overproducing cells. Proc. Natl. Acad. Sci. USA 91, 8930-8934.

Tang H., Ariki K., Ohkido M., Murakami Y., Matsufuji S., Li Z. H., and Yamamura K. I. (2009), Role of ornithine decarboxylase antizyme inhibitor in vivo. Genes Cells 14, 79-87.

Tohgo A., Eiraku M., Miyazaki T., Miura E., Kawaguchi S. Y., Nishi M., Watanabe M., Hirano T., Kengaku M., and Takashima H. (2006), Impaired cerebellar functions in mutant mice lacking DNER. Mol. Cell. Neurosci. 31, 326-333.

Tsuji T., Katsurano M., Ibaragi S., Shima K., Sasaki A., and $\mathrm{Hu}$ G. F. (2007), Ornithine decarboxylase antizyme upregulates DNA-dependent protein kinase and enhances the nonhomologous end-joining repair of DNA double-strand breaks in human oral cancer cells. Biochemistry 46, 8920-8932.

Wang Z., Hao Y., and Lowe A. W. (2008), The adenocarcinoma-associated antigen, AGR2, promotes tumor growth, cell migration, and cellular transformation. Cancer Res. 68, 492-497. 